PROCEEDINGS OF THE AMERICAN MATHEMATICAL SOCIETY

Volume 124, Number 11, November 1996

\title{
ON SPREAD AND CONDENSATIONS
}

\author{
A. V. ARHANGELSKII
}

(Communicated by Franklin D. Tall)

\begin{abstract}
A space $X$ has a property $\mathcal{P}$ strictly if every finite power of $X$ has $\mathcal{P}$. A condensation is a one-to-one continuous mapping onto. For Tychonoff spaces, the following results are established. If the strict spread of $X$ is countable, then $X$ can be condensed onto a strictly hereditarily separable space. If $s\left(C_{p}(X)\right) \leq \omega$, then $C_{p}(X)$ can be condensed onto a strictly hereditarily separable space, and therefore, every compact subspace of $C_{p}(X)$ is strictly hereditarily separable. Under $(M A+\neg C H)$, if $G$ is a topological group such that $s\left(C_{p}(G)\right) \leq \omega$, then $G$ is strictly hereditarily Lindelöf and strictly hereditarily separable.
\end{abstract}

All spaces considered in this paper are beforehand assumed to be Hausdorff, with the exception of the spaces $X_{p}$ and $Y_{p}$ (see the proof of Theorem 2). Under $a$ condensation we understand a one-to-one continuous mapping of a topological space onto a topological space. Which topological properties can be improved by means of condensations? Behind this general question one can distinguish many interesting concrete problems. In particular, to what extent can various compactness type properties be improved by condensations? We understand here compactness type properties in a very general way; we count among them not only compactness itself, countable compactness, pseudocompactness and Lindelöf property, but also separability, countable cellularity, and all other topological invariants which in the presence of discreteness make the space countable. Of course, in such matters we have to restrict ourselves with the class of Hausdorff spaces, since every $T_{1}$-space $X$ can be condensed onto a hereditarily separable compact $T_{1}$-space; it suffices to take the cofinite topology on $X$.

We would like to point at an interesting feature of the condensation problem. It is well known that for certain topological properties it is true that as soon as a space has the property, all compact subspaces of this space have a much stronger topological property. For example, if a space is metrizable, then all compact subspaces of this space are not only metrizable but have a countable base as well. If all points in a Hausdorff space $X$ are of the type $G_{\delta}$, then each compact subspace of $X$ is first countable. In this situation, taking into account that condensations of compact spaces are homeomorphisms, one might ask whether the explanation of the phenomenon might lie in the existence of a condensation of a space with the

Received by the editors April 7, 1995.

1991 Mathematics Subject Classification. Primary 54A25, 54C35, 54A35.

Key words and phrases. Spread, hereditary density, condensation, Lindelöf space, function spaces, topology of pointwise convergence, small diagonal, caliber.

The author was partially supported by NSF grant DMS-9312363.

(C)1996 American Mathematical Society 
weaker property onto a space with the stronger property. Of course, this argument would make sense only for closed hereditary properties.

In this article we present a few results on spread and condensations which show that the above conjecture sometimes comes to be true. Some closely related results from $C_{p}$-theory are also included. Among main results of this paper are Theorems $1,3,6,8,9$, and Corollary 5 .

Our terminology and notation are as in [3] and [10]. We say that a topological space has a property $\mathcal{P}$ strictly (or is strictly $\mathcal{P}$ ), if every finite power of $X$ has the property $\mathcal{P}$. By $C_{p}(X)$ we denote the space of all real-valued continuous functions on a Tychonoff space $X$ in the topology of pointwise convergence (see [1]). The spread $s(X)$ of a space $X$ is the smallest infinite cardinal number $\tau$ such that the cardinality of every discrete subspace of $X$ is not greater than $\tau$. A space $X$ is said to be left separated, or left, if there is a well ordering $<$ on $X$ such that all initial intervals with respect to $<$ are closed in $X$.

Proposition 1. Every regular (Tychonoff) space $X$ of strictly countable spread can be condensed onto a regular (Tychonoff) strictly hereditarily separable space.

Proof. It is well known (see, for example, [2]) that if the spread of the product $X \times Y$ is countable, then either $X$ is hereditarily separable or $Y$ is hereditarily Lindelöf. It follows that every space of strictly countable spread is either strictly hereditarily separable or strictly hereditarily Lindelöf. In the first case there is nothing to prove. In the second case, $X \times X$ is a hereditarily Lindelöf space, and, therefore, the diagonal in $X \times X$ is a $G_{\delta}$-set. It remains to recall that every regular Lindelöf space with the last property can be condensed onto a separable metrizable space [10].

Corollary 1. If the strict spread of a regular space $X$ is countable, then every compact subspace of $X$ is strictly hereditarily separable.

Example. It is consistent with $Z F C$ to assume that there is a strictly hereditarily separable compactum $X$, which is not hereditarily Lindelöf (see [15], [17]). Clearly, $X$ cannot be condensed onto a hereditarily Lindelöf space. Therefore, we cannot replace "strictly hereditarily separable" in Proposition 1 by "hereditarily Lindelöf". The free topological group $F(X)$ of $X$ is a $\sigma$-compact strictly hereditarily separable homogeneous space, which cannot be condensed onto a hereditarily Lindelöf space. This shows that the assumption $(M A+\neg C H)$ in Theorem 5 below is essential. Put $Y=C_{p}(X)$. Then $Y$ is strictly hereditarily Lindelöf, by Velichko-Zenor's theorem [21], [22], and the strict tightness of $Y$ is countable [1], while $Y$ is not separable, since $X$ cannot be condensed onto a separable metrizable space [1]. The space $C_{p}(Y)$ is strictly hereditarily separable, again by Velicko-Zenor's theorem, and it also cannot be condensed onto a hereditarily Lindelöf space, since it contains a topological copy of $X$. This shows that we cannot replace "strictly hereditarily separable" in Theorem 1 below with "hereditarily Lidelöf".

Theorem 1. If $X$ is a Tychonoff space such that the spread of $C_{p}(X)$ is countable, then $C_{p}(X)$ can be condensed onto a strictly hereditarily separable Tychonoff space.

In fact, we shall prove a slightly stronger assertion. Note that it is still unknown whether one can prove in $Z F C$ that if the spread of $C_{p}(X)$ is countable, then the strict spread of $C_{p}(X)$ is also countable (Velichko's problem); therefore, one cannot just deduce Theorem 1 from Proposition 1. 
If $Y$ is a subspace of a Tychonoff space $X$, then $C_{p}(Y \mid X)$ is the subspace of $C_{p}(Y)$ consisting of restrictions to $Y$ of elements of $C_{p}(X)$.

Proposition 2. For any subspace $Y$ of a Tychonoff space $X, s(Y \times Y) \leq$ $s\left(C_{p}(Y \mid X)\right)$.

The proof of Corollary 2.5.19 in [1] trivially transforms into a proof of Proposition 2. For a space $X$, let $X_{p}$ be the space of all finite subsets of $X$ in the $\kappa$-topology; the family of all sets of the form: $U^{*}=\{K: K \subset U,|K|<\omega\}$, where $U$ is any open subset of $X$, is a base of $X_{p}$.

Proposition 3. For any subspace $Y$ of a Tychonoff space $X$ the next inequality holds: $s\left(Y_{p}\right) \leq s\left(C_{p}(Y \mid X)\right)$.

Proof. Let $Z$ be a discrete subspace of $Y_{p}$. For each $K \in Z$ we can fix an open subset $U_{K}$ of $X$ so that $U_{K}{ }^{*} \cap Z$ contains only the point $K$. Now we can choose a continuous real-valued function $f_{K}$ on $X$ such that $f_{K}(K)=\{1\}$ and $f_{K}\left(X \backslash U_{K}\right)=\{0\}$. Then $F=\left\{f_{K}: K \in Z\right\}$ is a discrete subspace of $C_{p}(Y \mid X)$. Indeed, the set $V_{K}$ of all $f \in C_{p}(Y \mid X)$ such that $f(y)>0$ for each $y \in K$ is an open subset of $C_{p}(Y \mid X)$, and $V_{K} \cap F=\left\{f_{K}\right\}$. Therefore, $|Z| \leq|F| \leq s\left(C_{p}(Y \mid X)\right)$, which completes the proof.

An absolute version of Proposition 3 was formulated by M. Asanov in [9].

Theorem 2. Let $Y$ be a subspace of a Tychonoff space $X$ such that the spread of $C_{p}(Y \mid X)$ is countable. Then $C_{p}(Y)$ can be condensed onto a strictly hereditarily separable Tychonoff space.

Proof. By Proposition 2, $s(Y \times Y) \leq \omega$. There is a dense left subspace $Z$ in $Y$ (see [3]). Then $Z \times Z$ is a left space of the countable spread [4], and therefore, $Z \times Z$ is hereditarily Lindelöf [3], [16]. It follows that the diagonal in $Z \times Z$ is a $G_{\delta}$. By Proposition $3, s\left(Y_{p}\right) \leq s\left(C_{p}(Y \mid X)\right) \leq \omega$. Since $Z_{p}$ is obviously a subspace of $Y_{p}$, we have: $s\left(Z_{p}\right) \leq s\left(Y_{p}\right) \leq \omega$. By Asanov's theorem, since the spread of $Z_{p}$ is countable, and $Z$ is a space with $G_{\delta}$-diagonal, the strict spread of $Z$ is also countable [9]. Now, all finite powers of the space $Z$ are left spaces, since $Z$ is a left space [4]. It follows that the space $Z^{n}$ is hereditarily Lindelöf, for each $n \in \omega$ [3]. Thus, $Z$ is strictly hereditarily Lindelöf. Then, by Velichko-Zenor's theorem [21], [22], the space $C_{p}(Z)$ is strictly hereditarily separable. Obviously, the restriction mapping condenses $C_{p}(Y)$ onto a subspace $W$ of $C_{p}(Z)$, where $W$ is also strictly hereditarily separable.

Corollary 2. If $Y$ is a subspace of a Tychonoff space $X$ such that the spread of $C_{p}(Y \mid X)$ is countable, then every compact subspace of $C_{p}(Y)$ is strictly hereditarily separable.

Problem 1. Let $X$ be a regular hereditarily Lindelöf space. Is it true that $C_{p}(X)$ can be condensed onto a hereditarily separable (Tychonoff, regular) space?

Observe, that this question is closely related to the $L$-space problem. Indeed, if every regular hereditarily Lindelö space is separable, then the answer to Problem 1 is "yes", since then $C_{p}(X)$ can be condensed onto a separable metrizable space. This observation may be considered as a motivation of the problem. Note that the above proof of Theorem 2 also contains a proof of the next result: 
Theorem 3. If $Y$ is a subspace of a Tychonoff space $X$ such that the spread of $C_{p}(Y \mid X)$ is countable, then $Y$ contains a dense strictly hereditarily Lindelöf subspace.

We denote by $(M A+\neg C H)$ the combination of Martin's Axiom $M A$ with the negation of $\mathrm{CH}$. Under this assumption Theorem 2 can be considerably strengthened.

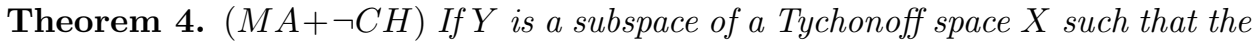
spread of $C_{p}(Y \mid X)$ is countable, then $C_{p}(Y)$ condenses onto a separable metrizable space, every pseudocompact subspace $F$ of $C_{p}(Y)$ is metrizable, and $Y$ is hereditarily separable and hereditarily Lindelöf.

Proof. By Theorem 2, $C_{p}(Y)$ condenses onto a strictly hereditarily separable Tychonoff space $Z$. By K. Kunen's theorem [14], $Z$ is strictly hereditarily Lindelöf. Therefore, $Z$ can be condensed onto a separable metrizable space [7], [10]. Since every one-to-one continuous mapping of a pseudocompact Tychonoff space onto a metrizable space is a homeomorphism [10], it follows that $F$ is metrizable. For every subspace $H$ of $Y$, the spread of $C_{p}(H \mid X)$ is countable, since $C_{p}(H \mid X)$ is a continuous image of $C_{p}(Y \mid X)$. Therefore, to show that $Y$ is hereditarily separable it suffices to prove that $Y$ itself is separable. By Theorem 3, there is a dense strictly hereditarily Lindelöf subspace $Z$ in $Y$. Under $(M A+\neg C H) Z$ has to be separable, by a theorem of $\mathrm{K}$. Kunen [14]. It follows that $Y$ is separable, and hence, hereditarily separable. Now we can apply a version of Theorem 19 from [20], which instead of $C_{p}(X)$ refers to $C_{p}(Y \mid X)$ (the proof of Theorem 19 in [20] is valid in this case as well). By this result, since $Y$ is hereditarily separable, it has to be hereditarily Lindelöf.

Theorem 5. ( $M A+\neg C H)$ If $G$ is a topological group such that the spread of $C_{p}(G)$ is countable, then the space $G$ is strictly hereditarily separable and strictly hereditarily Lindelöf.

Proof. By Theorem 4, G is hereditarily Lindelöf. Therefore, the pseudocharacter of the space $G$ is countable. Since $G$ is a topological group, the space $G$ can be condensed onto a metrizable space (see [10]). From Asanov's theorem [9] it follows that the strict spread of $G$ is countable. This implies that either $G$ is strictly hereditarily separable or $G$ is strictly hereditarily Lindelöf [2]. Then, by Kunen's theorem [14], $G$ is strictly hereditarily separable and strictly hereditarily Lindelöf.

Some of our results can be considerably strengthened if we impose additional restrictions on $X$. Recall that a space $X$ is $\omega$-monolithic [1] if for each countable subset $A$ of $X$ the networkweight of the subspace $\bar{A}$ is countable. A Tychonoff space $X$ is said to be $\omega$-stable [1], if every Tychonoff continuous image $Y$ of $X$ such that there is a condensation of $Y$ onto a separable metrizable space has a countable network.

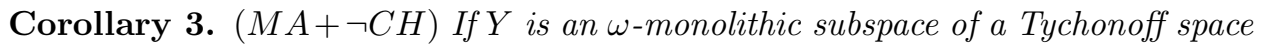
$X$, and the spread of $C_{p}(Y \mid X)$ is countable, then $Y$ and $C_{p}(Y)$ have a countable network.

Proof. Since $Y$ is $\omega$-monolithic, $C_{p}(Y)$ is $\omega$-stable [1]. Now it follows from Theorem 4 that $C_{p}(Y)$ has a countable network. Then $Y$ also has a countable network [1]. 
One cannot omit the assumption $(M A+\neg C H)$ in Theorem 4 and Corollary 3 (see Example 1); however, we can prove a slightly weaker assertion:

Theorem 6. If $X$ is a Tychonoff $\omega$-monolithic space such that the spread of $C_{p}(X)$ is countable, then $X$ is strictly hereditarily Lindelöf, and $C_{p}(X)$ is strictly hereditarily separable.

Proof. We need a preliminary result. Let us call a space $X$ weakly $\omega$-monolithic, if every subspace $Y$ of $X$ such that there is a countable dense discrete subspace $Z$ in $Y$, is Lindelöf.

Proposition 4. Every weakly $\omega$-monolithic space $X$ of the countable spread is hereditarily Lindelöf.

Proof. Let $\gamma$ be any family of open subsets of $X$. It is enough to show that there is a countable subfamily $\eta$ of $\gamma$ such that $\cup \gamma=\cup \eta$. By the well known Shapirovskij's Lemma [18], one can find a countable subfamily $\xi \subset \gamma$ and a countable discrete subspace $A \subset \cup \gamma$ such that $\cup \gamma \subset \cup \xi \cup \bar{A}$. Since $X$ is weakly $\omega$-monolithic, the subspace $Y=\bar{A} \cap(\cup \gamma)$ is Lindelöf. Therefore, there is a countable subfamily $\lambda$ of $\gamma$ such that $Y \subset \cup \lambda$. Put $\eta=\xi \cup \lambda$. Then $\eta$ is a countable subfamily of $\gamma$ such that $\cup \eta=\cup \gamma$.

We continue the proof of Theorem 6 . The spread of $X \times X$ is countable [1], and $X \times X$ is also $\omega$-monolithic. By Proposition 4 , it follows that $X \times X$ is hereditarily Lindelöf. This implies that $X$ has a $G_{\delta}$-diagonal. Then, by Asanov's theorem, the strict spread of $X$ is countable. Since all finite powers of $X$ are $\omega$-monolithic, it follows from Proposition 4 that $X$ is strictly hereditarily Lindelöf. Then, by Velichko-Zenor's theorem, $C_{p}(X)$ is strictly hereditarily separable.

Problem 2. Let $X$ be a Tychonoff space, and let $Y$ be a subspace of $X$. Is then $s(Y)$ equal to $s(Y \mid X)$ ?

The answer to this very natural question is not known to the author at present; however, a partial result, based on a special assumption, is available.

Let us denote by $(S)$ the next assertion: every Tychonoff hereditarily separable space is Lindelöf. S. Todorčević has shown [19] that $(S)$ is consistent with $Z F C$.

Theorem 7. (S) Let $Y$ be a subspace of a Tychonoff space $X$. Then the spread of $C_{p}(Y \mid X)$ is countable if and only if the spread of $C_{p}(Y)$ is countable.

Proof. Since $C_{p}(Y \mid X)$ is a subspace of $C_{p}(Y)$, the spread of $C_{p}(Y \mid X)$ does not exceed the spread of $C_{p}(Y)$. Let us now assume that the spread of $C_{p}(Y \mid X)$ is countable. By Proposition 2, $s(Y \times Y) \leq \omega$. Therefore, every right separated subspace of $Y \times Y$ is hereditarily separable (see [16]), and it now follows from $(S)$ that every right separated subspace of $Y \times Y$ is hereditarily Lindelöf, and hence, countable. Thus, $Y \times Y$ is hereditarily Lindelöf, which implies that $Y$ has a $G_{\delta^{-}}$ diagonal. By Proposition 3, we also have: $s\left(Y_{p}\right) \leq \omega$. From Asanov's theorem it now follows that the strict spread of $Y$ is countable. As we have noted above, $(S)$ now implies that $Y$ is strictly hereditarily Lindelöf. Then, by Velichko-Zenor's theorem, $C_{p}(Y)$ is strictly hereditarily separable; (therefore, by $(S), C_{p}(Y)$ is also strictly hereditarily Lindelöf, and $Y$ is strictly hereditarily separable; however, such implications are already known-see [2]). 
Corollary 4. (S) If $X$ is a Tychonoff space such that the spread of $C_{p}(X)$ is countable, then for every subspace $Y$ of $X$ the spread of $C_{p}(Y)$ is countable.

Proof. Clearly, $s\left(C_{p}(Y \mid X)\right) \leq s\left(C_{p}(X)\right)$, for every subspace $Y$ of $X$. It remains to apply Theorem 7 .

A crucial role in many arguments above was played by Asanov's theorem [9]. It seems worth noting that this theorem can be strengthened in a natural way. Recall, that $X$ is a space with a small diagonal, if for every uncountable subset $A$ of $X \times X$ disjoint from the diagonal $\Delta_{X}=\{(x, x): x \in X\}$ there is an open neighbourhood $U$ of $\Delta_{X}$ in $X \times X$ such that the set $A \backslash U$ is uncountable. This delicate notion was introduced by M. Hušek in [13]. Clearly, every space with a $G_{\delta}$-diagonal is also a space with a small diagonal, but the converse is not true (see [8]). Therefore, the next theorem is stronger than Asanov's theorem.

Theorem 8. If $Y$ is a subspace of a Tychonoff space $X$ such that the spread of $C_{p}(Y \mid X)$ is countable, and $Y$ has a small diagonal, then the strict spread of $C_{p}(Y)$ is countable.

The proof relies heavily on Asanov's argument. For the sake of completeness, we present it here in detail; all the more so, since Asanov's paper where the proof was published, is not only out of reach of many topologists, but also contains a few discrepancies. First, some notation. For a space $X$ and $n \in \omega$, we denote by $\Psi_{n}$ the natural mapping of the space $X^{n}$ into the space $X_{p}$. The mapping $\Psi_{n}$ is obviously continuous and finite-to-one. We also denote by $\Delta_{X^{n}}$ the set of all points $\left(x_{1}, \ldots, x_{n}\right)$ of $X^{n}$ at least two coordinates of which coincide. We call this subspace of $X^{n}$ the diagonal of $X^{n}$.

Lemma 1. For each open neighbourhood $U$ of $\Delta_{X^{n}}$ in $X^{n}$ there is an open neighbourhood $V$ of $\Delta_{X^{n}}$ in $X^{n}$ such that $V \subset U$, and the restriction of $\Psi_{n}$ to $X^{n} \backslash V$ is an open continuous finite-to-one mapping onto a subspace of $X_{p}$.

Proof. Let $S_{n}$ be the group of all permutations on $\{1, \ldots, n\}$. This group acts naturally on $X^{n}$, and for $s \in S_{n}$ let $s(U)$ be the image of $U$ under $s$. Put $V=$ $\cap\left\{s(U): s \in S_{n}\right\}, Y=X^{n} \backslash V$, and $Z=\Psi_{n}(Y)$. Clearly, the restriction of $\Psi_{n}$ to $Y$ is a finite-to-one continuous mapping of $Y$ onto $Z$. Let us show that this mapping is open.

Fix a point $\left(x_{1}, \ldots, x_{n}\right)$ in $Y$. Since this point is not in $\Delta_{X^{n}}$, there are open sets $O_{1}, O_{2}, \ldots, O_{n}$ in $X$ such that $x_{i} \in O_{i}$ for each $i \in\{1, \ldots, n\}, O_{i} \cap O_{j}=\emptyset$ if $i \neq j$, and the following condition is satisfied:

(1) if $\left\{V_{1}, \ldots, V_{n}\right\}$ is a proper subset of $\gamma=\left\{O_{1}, \ldots, O_{n}\right\}$, then $V_{1} \times \ldots \times V_{n} \subset V$. Put $O=O_{1} \times \ldots \times O_{n}, G=O_{1} \cup \ldots \cup O_{n}$, and $W=\{A \in Z: A \subset G\}$. To prove that the mapping $\Psi_{n}$ restricted to $Y$ is open it suffices to show that $\Psi_{n}(O \cap Y)=W$, since $O \cap Y$ can be treated as a basic open set in $Y$, and $W$ is open in $Z$. For brevity, put $H=\Psi_{n}(O \cap Y)$. The inclusion $H \subset W$ is obvious. Let us prove that $W \subset H$. Take any $A \in Z$ such that $A \subset G$. The set $A$ consists of exactly $n$ points, since it is in $\Psi_{n}\left(X^{n} \backslash \Delta_{X^{n}}\right)$. Since $A \in Z$, there is $a=\left(a_{1}, \ldots, a_{n}\right) \in Y$ such that $\Psi_{n}(a)=A$, that is, $\left\{a_{1}, \ldots, a_{n}\right\}=A$. The family $\gamma=\left\{O_{1}, \ldots, O_{n}\right\}$ is disjoint and consists of exactly $n$ elements. If $A \cap O_{i} \neq \emptyset$ for each $i \in\{1, \ldots, n\}$, fix $y_{i} \in O_{i} \cap A$. Clearly, $A=\left\{y_{i}: i=1, \ldots, n\right\}, y=\left(y_{1}, \ldots, y_{n}\right) \in O$, and $\Psi_{n}(y)=A$. Let us show that $y \in Y$. From $|A|=n$ it follows, that $a$ is obtained by a permutation $s$ from $y$. Therefore, if $y$ were in $V$, then $a$ would be in $V$, in 
contradiction with $a \in Y=X^{n} \backslash V$. Thus, $y \in Y$. Let us now consider the case when, for some $j \in\{1, \ldots, n\}, O_{j} \cap A=\emptyset$. For $i \in\{1, \ldots, n\}$ let $V_{i}$ be any member of $\gamma$ such that $a_{i} \in V_{i}$. Clearly, $\left\{V_{1}, \ldots, V_{n}\right\}$ is a proper subfamily of $\gamma$, therefore, $a \in V_{1} \times \ldots \times V_{n} \subset V$, contradicting to $a \in Y=X^{n} \backslash V$. Thus, the second case is impossible, and $y \in Y$. It follows that $y \in O \cap Y$, and $A=\Psi_{n}(y) \in H$; hence, $H=W$.

Lemma 2. Let $f$ be an open continuous finite-to-one mapping of a space $Y$ onto a space $Z$. Then $s(Y)=s(Z)$.

Proof. Since $f(Y)=Z, s(Z) \leq s(Y)$. Let us show that $s(Y) \leq s(Z)$. Take any discrete subset $D$ of $Y$. Without any loss in generality, we can assume that $|D|$ is not countably cofinal. For each $x \in D$ fix an open neighbourhood $O_{x}$ of $x$ in $Y$ such that $O_{x} \cap D=\{x\}$, and put $M=\cup\left\{O_{x}: x \in D\right\}$. Let $\phi$ be the restriction of $f$ to $M$. Clearly, $D$ is closed in $M$. There are $k \in \omega$ and $B \subset D$ such that $\left|\phi^{-1} \phi(y)\right|=k$ for each $y \in B$, and $|B|=|D|$. Put $P=\phi^{-1} \phi(B)$. Then $B \subset P \subset M$, and $B$ is closed in $P$, since $B$ is closed in $M$. The mapping $\phi$ is open, since $M$ is open in $Y$. Therefore, the restriction of $\phi$ to $P$ is an open mapping of $P$ onto $\phi(P)=\phi(B)$ (see [10]). Let us denote this mapping by $g$. The mapping $g$ is also continuous and exactly $k$-to-one. Then $g$ is a closed mapping [7], which implies that $\phi(A)$ is closed in $\phi(B)$ for each $A \subset B$. Thus, $\phi(B)$ is a discrete subspace of $Z$ of the same cardinality as $B$ and $D$; it follows that $|D| \leq s(Z)$ and $s(Y) \leq s(Z)$.

For a cardinal number $\tau$, let us say that a space $X$ has a $\tau$-small diagonal, if for each $A \subset\left(X \times X \backslash \Delta_{X}\right)$ such that $\tau<|A|$ there is an open neighbourhood $U$ of $\Delta_{X}$ in $X \times X$ such that $\tau<|A \backslash U|$. If the above condition is satisfied with $X \times X$ replaced by $X$ and $\Delta_{X}$ replaced by a subset $P$ of $X$, we say that $P$ is $\tau$-small in $X$.

For a space $X$ and $n \in \omega$ we put $X_{p, n}=\left\{A \in X_{p}:|A|=n\right\}$.

Proposition 5. Let $X$ be a space with a small diagonal, and let $s\left(X_{p, n}\right) \leq \omega$ for some $n \in \omega$. Then $s\left(X^{n} \backslash U\right) \leq \omega$ for each open neighbourhood $U$ of the diagonal $\Delta_{X^{n}}$ in $X^{n}$ (for the same $n$ ).

Proof. This follows from Lemma 1 and Lemma 2, since the spread of a subspace is always not greater than the spread of the whole space. We also take into account that $\Psi_{n}$ maps $X^{n} \backslash \Delta_{X^{n}}$ into $X_{p, n}$.

Of course, a general version of Theorem 8 implies that if $X$ is a Tychonoff space with a $\tau$-small diagonal, and $s\left(C_{p}(X)\right) \leq \tau$, then $s\left(X^{n}\right) \leq \tau$ and $s\left(\left(C_{p}(X)^{n}\right) \leq \tau\right.$, for each $n \in \omega$. Since the argument in the general case remains the same, we just prove Theorem 8 .

Proof of Theorem 8. By Proposition 3, $s\left(Y_{p}\right) \leq s\left(C_{p}(Y \mid X)\right) \leq \omega$. In particular, $s(Y) \leq \omega$. This brings us into a position to apply induction on $n$.

Fix $n \in \omega$, and assume that $s\left(Y^{n-1}\right) \leq \omega$. Take any discrete subspace $D$ of $Y^{n}$. We have to show that $D$ is countable. Assume the contrary. Put $D_{1}=D \cap \Delta_{Y^{n}}$, and $D_{2}=D \backslash D_{1}$. It is clear that $\Delta_{Y^{n}}$ is the union of a finite number of topological copies of the space $Y^{n-1}$; therefore, $\left|D_{1}\right| \leq \omega$. It follows that $D_{2}$ is uncountable. Since $\Delta_{Y}$ is $\omega$-small, $\Delta_{Y^{n}}$ is $\omega$-small in $Y^{n}$-this was shown in [8]. Therefore, there is an open neighbourhood $U$ of $\Delta_{Y^{n}}$ in $Y^{n}$ such that $D_{U}=D_{2} \backslash U$ is uncountable. On the other hand, $s\left(Y^{n} \backslash U\right) \leq s\left(Y_{p}\right) \leq \omega$, by Proposition 5, which implies that $D_{U}$ is countable, since $D_{U}$ is a discrete subspace of $Y^{n} \backslash U$. 
Theorem 9. If $X$ is a Tychonoff space such that $\aleph_{1}$ is a caliber of $C_{p}(X)$, and the spread of $C_{p}(X)$ is countable, then the strict spread of $C_{p}(X)$ is also countable.

Proof. A Tychonoff space $X$ has a small diagonal if and only if $\aleph_{1}$ is a caliber of $C_{p}(X)$, by a result in [8]. It remains to apply Theorem 8 .

It was shown in [5] that if a Tychonoff space is cleavable, then it has a small diagonal. Combining this characterization with Theorem 8 , we arrive at the next conclusion:

Corollary 5. If $X$ is a cleavable Tychonoff space such that $s\left(C_{p}(X)\right) \leq \omega$, then the strict spread of $C_{p}(X)$ and of $X$ is countable.

Observe that, according to a basic property of a cleavable Tychonoff space, we can reformulate Corollary 5 as follows: if $s\left(C_{p}(X)\right) \leq \omega$, and $R^{X}=\cup\{\bar{A}: A \subset$ $\left.C_{p}(X),|A| \leq \omega\right\}$, then the strict spread of $C_{p}(X)$ is countable.

Problem 3. Is it true in $Z F C$ that every perfect hereditarily separable regular (or Tychonoff) space can be condensed onto a regular Lindelöf space? Onto a realcompact space?

Observe, that if we drop "perfect" in Problem 3 above, then the answer is "no", as one can see from the next example, discussed in detail in [6]. Under $(\mathrm{CH})$, there is a strictly hereditarily separable, strictly countably compact topological group $G$ which is not compact [11], [12]. The space $G$ is just what we need. Indeed, $G$ cannot be condensed onto a realcompact space since such a space would have been a hereditarily separable dyadic compactum, and therefore, would have been metrizable. See the details in [6].

Problem 4. Is it true that every Tychonoff space $X$ can be condensed onto a Tychonoff space $Y$ such that every discrete family of non-empty open subsets in $Y$ is countable?

Clearly, all the results above which were obtained without special set-theoretic assumptions remain true not only in the countable case - they hold for any infinite cardinal number $\tau$.

Remark. The proof of Theorem 7 shows that the next assertions A and B are equivalent in $Z F C$ :

A. Every hereditarily separable Tychonoff (regular) space is Lindelöf.

B. Every Tychonoff (regular) space of the countable spread is Lindelöf.

Some topologists would recognize this fact as a folklore. The equivalence implies that under $(S)$ every pseudocompact Tychonoff space of the countable spread is compact and the tightness of it is countable.

\section{REFERENCES}

[1] Arhangel'skii A.V., Topological function spaces, vol. 78, Dordrecht, Kluwer Academic Publishers, Mathematics and its Applications, 1992, (translated from Russian). MR 92i:54022

[2] Arhangel'skii A.V., Hereditarily Lindelöf spaces of continuous functions, Moscow Univers. Math. Bull. 44 (1989), 67-69. MR 90m:54007

[3] Arhangel'skii A.V., Structure and classification of topological spaces and cardinal invariants, Russian Math. Surveys 33 (1978), no. 6, 33-96. MR 80i:54005

[4] Arhangel'skii A.V., Left-expanded spaces, Mosc. Univ. Math. Bull. 32 (1977), 22-27. MR 58:2746 
[5] Arhangel'skii A.V. and A. Bella, A few observations on topological spaces with small diagonal, Zbornik radova Filozofskog faculteta u Nišu Ser. Mat. 6 (1992), 211-213. CMP 1994:3

[6] Arhangel'skii A.V. and Fedorchuk V.V., On condensations of countably compact spaces onto compacta, Fund. Prikl. Mat. 1 (1995), 871-880. (Russian)

[7] Arhangel'skii A.V. and Ponomarev V.I., Fundamentals of General Topology: problems and exercises, Reidel, 1984, (translated from Russian). MR 87i:54001

[8] Arhangel'skii A.V. and Tkachuk V.V., Calibers and point-finite cellularity of the spaces $C_{p}(X)$, and some questions of $S$. Gul'ko and $M$ Hušek, Topology and Appl. 23 (1986), 65-74. MR 87h:54036

[9] Asanov M., Cardinal invariants of spaces of continuous functions, Modern Topology and Set Theory, Izhevskij Universitet, Izhevsk, 1979, pp. (8-12). (Russian)

[10] Engelking R., General Topology, PWN, Warsaw, 1977. MR 58:18316b

[11] Hajnal A. and I. Juhasz, On hereditarily $\alpha$-Lindelöf and $\alpha$-separable spaces, 2, Fund. Math. 81 (1974), 147-158. MR 49:1478

[12] Hajnal A. and I. Juhasz, A separable normal topological group need not be Lindelöf, General Topology and Appl. 6 (1976), 199-205. MR 55:4088

[13] Hušek M., Topological spaces without $\kappa$-accessible diagonal, Comm. Math. Univ. Carol. (1977), 777-788. MR 58:24198

[14] Kunen K., Strong $S$ and L spaces under MA, Set-theoretic Topology (G.M. Reed, ed.), Academic Press, New-York, 1977, pp. 265-268. MR 55:13362

[15] Negrepontis S., Banach Spaces and Topology, Handbook of Set-theoretic Topology (K. Kunen and J.E. Vaughan, eds.), North-Holland, Amsterdam, 1984, pp. (1047-1142). MR 86i:46018

[16] Roitman J., The spread of regular spaces, General Topology and Appl. 8 (1978), 85-91. MR 58:12909

[17] Roitman J., Basic $S$ and L, Handbook of Set-theoretic Topology (K. Kunen and J.E. Vaughan, eds.), North-Holland, Amsterdam, 1984, pp. (295-326). MR 87a:54043

[18] Shapirovskij B.E., On discrete subspaces of topological spaces. Weight, tightness and Souslin number, Soviet Math. Dokl. 13 (1972), 215-219. MR 45:1100

[19] Todorčević S., Forcing positive partition relations, Trans. Amer. Math. Soc. 280 (1983), 703-720. MR 85d:03102

[20] Todorčević S., Some Applications of $S$ and L Combinatorics, Annals of the New York Academy of Sciences 705 (1993), 130-167. MR 95j:54006

[21] Velichko N.V., Weak topology of spaces of continuous functions, Math. Notes 30 (1981), 849-854. MR 83f:54006

[22] Zenor P., Hereditary $m$-separability and the hereditary $m$-Lindelöf property in product spaces and function spaces, Fundam. Math. 106 (1980), 175-180. MR 82a:54039

Chair of General Topology and Geometry, Mech.-Math. Faculty, Moscow UniverSity, Moscow 119899, Russia (June 15-December 31)

Department of Mathematics, 321 Morton Hall, Ohio University, Athens, Ohio 45701 (JANUARY 1-JUNE 15)

E-mail address: aarhange@oucsace.cs.ohiou.edu 